\title{
Agustin ("Tino") Castellanos Jr. (1927-2017): The Journal Editors honor the grand legacy of a gentleman and a scholar
}

\author{
Sanjeev Saksena ${ }^{1} \cdot$ Samuel Levy ${ }^{2}$ \\ Published online: 8 August 2018 \\ (C) Springer Science+Business Media, LLC, part of Springer Nature 2018
}

This special issue of the Journal is a tribute and dedicated to the memory and contributions of a legendary figure in the field of cardiac electrophysiology, Professor Agustin Castellanos Jr. The history of the development of this medical discipline is a tale of great and inspiring contributions made by individuals who were simultaneously innovators and human beings with great personal character. In telling the story of this field, we may or may not fully appreciate their times, the environment, and the challenges that these researchers experienced. In issues such as this one, not only we can bring out these aspects of the honoree but also chart out the subsequent development and current status of knowledge that evolved from these pioneering efforts.

Agustin ("Tino") Castellanos Jr. was born in Havana, Cuba in 1927 to a renowned pediatric cardiologist, Agustin Wilfredo Castellanos and his wife, Angela Sanchez de Castellanos. Following in the footsteps of his father, a pioneer of angiocardiography and nominee for the Nobel Prize in Medicine, he completed his medical studies at the University of Havana School of Medicine in 1953.Even at this early stage in his career, he had developed a strong interest in electrocardiographic research, and his early work focused on the infant heart and its electrical behavior. In 1960, he emigrated to the USA and joined the faculty of the University of Miami School of Medicine in 1962, where he researched, taught, and trained cardiologists and electrophysiologists from four continents over a period of six decades. Vectorcardiography was an early tool that provided him the ability to examine the electrophysiological behavior of the human heart in a three-dimensional framework, and he used it to examine the normal and the

Sanjeev Saksena cmenj@aol.com

1 Rutgers Robert Wood Johnson Medical School, Piscataway, NJ, USA

2 University of Aix-Marseille, Marseille, France diseased conduction system [1]. Studies of the conduction system led to his contributions to the understanding of bundle branch blocks and hemiblocks, especially during and after evolving acute myocardial infarction His monograph on this subject became a reference text [2]. He became an early adopter of the then experimental technique of intracardiac transcatheter electrical recordings, utilizing another tool to decipher mechanisms of cardiac arrhythmias, particularly supraventricular tachycardias. He pioneered the application of physiologic electrical principles to the early stages of development of the cardiac pacemaker. With his colleague Louis Lemberg, he reported the first implant of a demand ventricular pacemaker in a human being [3]. With Barouh Berkovits, he pioneered and laid the foundations for the physiologic behavior of the modern single and dual chamber pacemakers [4]. He applied transcatheter pacing to the diseased His Bundle in man in another pioneering stride towards physiologic human pacemaking. His early work identified both the potential and pitfalls of His Bundle pacing such as distal His- Purkinje block and Wenckebach phenomena during this pacing method [5]. In farsighted publications, he highlighted the syndromes of automatic ventricular arrhythmias, then labeled electrocardiographically as ventricular "parasystole", presaging our now burgeoning field of triggered ventricular arrhythmias of both benign and malignant nature [6]. When the posterior anatomic location of the slow pathway in AV nodal reentrant tachycardia was first described in man by Sung and colleagues, Castellanos was the unheralded self-effacing guide and mentor for this research. In later years, [7] Castellanos continued his studies of the evolution of human cardiac electrical functions in mammals with studies on the whale heart and had a keen interest in evolving therapies such as antitachycardia pacing, cardiac ablation, and antiarrhythmic drugs.

Most of his colleagues in Miami over a three decade period were or became icons in arrhythmology in their own right, but "Tino" Castellanos remained the permanent "heart and soul" of this school of electrophysiology (Fig. 1). For his generations of students, Tino was not only a master teacher but also 


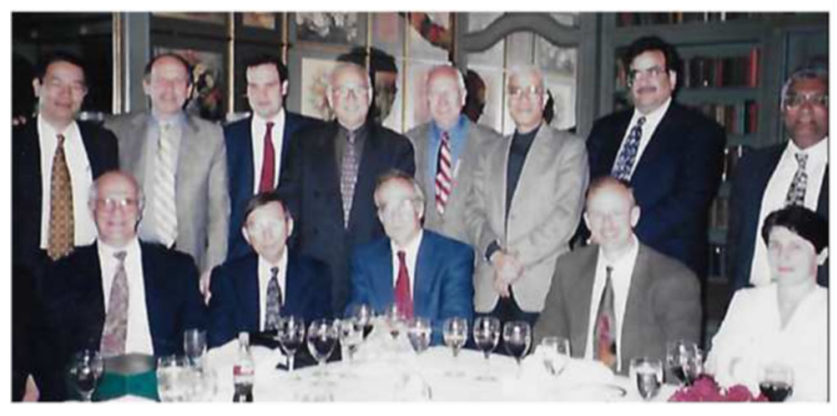

Fig. 1 Professor Agustin Castellanos Jr. is honored at a reunion of the University of Miami School of Electrophysiology in Toronto, Canada, 1999. Present are some of the University of Miami faculty and their electrophysiology trainees from 1972 to 1980 , who went on to establish their own electrophysiology training programs in the USA and Europe. Rear row standing: Ruey J. Sung MD, Richard Luceri MD, Raul Mitrani MD, Samuel Levy MD, Nabil El-Sherif MD, Ed Berbari PhD, Sanjeev Saksena MD. Front row sitting: Ralph Lazzara MD, Agustin Castellanos Jr. MD, Robert J. Myerburg MD, Nadir Saoudi MD, Gioa Turitto MD

an extraordinary example of humanity, humility, and selflessness. He trained innumerable students of electrophysiology and cardiology in his career, each with his or her own story of his impact on their lives. For the two of us, his actions and legacy influenced our careers profoundly, set the directions of our careers and made us into better physicians, researchers, and persons. In this introduction, we will take the opportunity to share two such stories briefly.

My co-editor Samuel Levy painted a tableau of this great man:

Dr Castellanos Jr. died in August 17, 2017 at age 89. With him we lost a "Giant" in the field of electrophysiology and cardiac arrhythmias. He was a fascinating scientist, a mentor to many colleagues and a gentleman. Those who had the chance to meet "Tino" will never forget him. He professed at the University of Miami from 1962 coming from Cuba to 2011. As he had his family in Miami, he did not accept the fabulous offers to join other prestigious Universities. Personally, I came to the University of Miami in 1971 with the intention to specialize in interventional cardiology at a time where clinical electrophysiology was in its infancy limited to His bundle recordings and electrical stimulation. Miami seemed to me the "Mecca of arrhythmology" with Benjamin Scherlag, Ralph Lazzara, Onkar Narula, Robert Myerburg, Nabil El Sherif, and of course Tino Castellanos. Very quickly, I was amazed by Tino Castellanos who used both "deductive reasoning" and a creative imagination. That was for me a fantastic opportunity to learn from him both arrhythmias and the principles which guided his life as he was also a philosopher. He was not interested by power, money or any form of advancement. He was a pure scientist, one that United States can be proud to have in any university.
There was no aspect of cardiac arrhythmias and of cardiac pacing that did not interest him and he wrote on various aspects of arrhythmia mechanisms. He admired his friends, electrocardiographers like Alfred Pick and Richard Langendorf, who imagined concepts such as "concealed conduction" only on the only basis of surface ECG, and became naturally their friend. He made numerous contributions and we will name only a few. With Barouh Berkovitz, another genius and Louis Lemberg, a remarkable cardiologist and clinician, they invented and implanted the first VVI Pacemaker in man. Tino Castellanos was the first to understand and describe the mechanism of circus movement tachycardias in patients with the Wolff-Parkinson White syndrome. He shared with me his interest for the "hemiblocks" concept of Mauricio Rosenbaum and described the diagnostic criteria of these conduction disturbances in acute myocardial infarction. He was also a fantastic teacher, a "Master", a mentor for many fellows seduced by his passion for science, for ECG interpretation and he knew how to transmit to his pupils his genuine enthusiasm. He had a major influence on my orientation and interest in the field of clinical electrophysiology on our life, a teacher with a great generosity. I was fortunate to have met him, to have met his family and his wife Marita, and to have been his friend. We shared so many pleasant moments together.

My own story is equally vintage "Tino". I came to the University of Miami in 1978 with a strong interest in myocardial mechanics and methods to measure and limit infarct size. Few shared my passion in this hallowed school of electrophysiology, but Tino in his quintessential way found a research project to keep my interest and connect it to his area of study. In a far sighted suggestion, he proposed addressing a major electrocardiographic problem of the time, namely the diagnosis of acute myocardial infarction in patients with left bundle branch block in an era without routine use of cardiac enzyme titers. We hypothesized that evolution of new regions of myocardial dyskinesis could confirm the diagnosis of acute myocardial infarction in patients with left bundle branch block. Key to this effort was to validate a regional wall motion technique analysis with gated blood pool angiography, which has developed into the current algorithms for this purpose in radionuclide angiography. In 1978, few imagined that the importance of myocardial dyskinesis in left bundle branch block would provide future directions for heart failure therapy. In later years when I was choosing both a location and direction of my career in this field, Tino's interest and caring for his trainees came to the forefront. His personal engagement helped us make important career choices. He arranged for an entire set of electrophysiological equipment to be delivered to my alma mater in India, All India Institute of Medical 
Sciences, to offer me the option of returning to my home in New Delhi. Simultaneously, he connected me to the premier cardiac institutions in New Jersey to offer me another alternative to consider. I tried out both, established the first comprehensive electrophysiology program and stayed the course in the latter for almost four decades. At my last visit with him in Miami several years ago, I came to pay my respects, but he would have none of it, and immediately corralled me to conduct ECG conference for the residents and fellows.

This special issue of the Journal is indeed a special and unique effort to bring physiologic concepts, current state of the art information, and novel research avenues in the area of Professor Castellanos' first love, the Cardiac Conduction system and Bundle Branch Blocks. In doing this, we hope to enshrine his contributions, remember his legacy, and provide new generations of electrophysiologists insights into the past, the present, and the future. To make this extraordinary issue happen, 17 editors of JICE, in conjunction with their colleagues, contributed original material to review and identify advances in this arena. We extend our special thanks to Dr. Castellanos' long standing colleague, collaborator, and alter ego, Dr. Robert Myerburg for his special insights in to the man and his career. We hope this memorable issue will make for an indelible memory of this remarkable human being.

\section{References}

1. Mayer JW, Lemberg L, Castellanos A Jr. The spatial vectorcardiogram in peri-infarction block. Am J Cardiol. 1963;11: 613-21.

2. Castellanos A Jr, Myerburg RJ. The hemiblocks in myocardial infarction. New York: Published by Appleton Century Crofts; 1976.

3. Castellanos A Jr., Lemberg L, Gosselin A: The demand cardiac pacemaker: a new instrument for the treatment of A-V conduction disturbances. Presented at the 7th inter American congress of cardiology, Montreal, June 1984.

4. Castellanos A Jr, Lemberg L, Jude JR, Mobin-Uddin K, Berkovits BV. Implantable demand pacemaker. Br Heart J. 1968 Jan;30(1):29-33.

5. Castellanos A, Moleiro F, Sung RJ, Myerburg RJ. Second degree His-Purkinje block during his bundle pacing. Am J Cardiol. 1978;41(6):1110-4.

6. Castellanos A Jr, Mayer JW, Lemberg L. Intermittent parasystole with disturbance in impulse formation and impulse conduction. Acta Cardiol. 1962;17:49-58.

7. Sung RJ, Waxman HL, Saksena S, Juma Z. Sequence of retrograde atrial activation in patients with dual atrioventricular nodal pathways. Circulation. 1981;64(5):1059-67. 